\title{
ARTICLE
}

Clinical Study

\section{A multicentre, prospective study of plasma circulating tumour DNA test for detecting $R A S$ mutation in patients with metastatic colorectal cancer}

\author{
Hideaki Bando ${ }^{1}$, Yoshinori Kagawa ${ }^{2}$, Takeshi Kato ${ }^{3}$, Kiwamu Akagi ${ }^{4}$, Tadamichi Denda ${ }^{5}$, Tomohiro Nishina ${ }^{6}$, Yoshito Komatsu ${ }^{7}$, \\ Eiji Oki $\mathbb{D}^{8}$, Toshihiro Kudo ${ }^{9}$, Hiroshi Kumamoto ${ }^{10}$, Takeharu Yamanaka ${ }^{11}$ and Takayuki Yoshino ${ }^{1}$
}

BACKGROUND: OncoBEAM ${ }^{\mathrm{TM}}$ RAS CRC kit using BEAMing technology is a circulating tumour DNA (ctDNA) test for detecting plasma RAS mutational status in metastatic colorectal cancer ( $\mathrm{mCRC}$ ). We conducted a multicentre, prospective study to investigate the concordance of the RAS mutational status between plasma ctDNA and tumour tissue DNA.

METHODS: $m C R C$ patients without prior anti-EGFR antibodies or regorafenib treatment were enroled. Plasma- and tissue-based RAS mutational status were determined by BEAMing, respectively.

RESULTS: A total of 280 patients from eight institutions were eligible. The overall agreement between plasma- and tissue-based analyses was $86.4 \%$, with a positive percent agreement of $82.1 \%$ and negative percent agreement of $90.4 \%$. From logistic regression analysis, lung metastasis alone indicated the most significant factor associated with discordance. The agreement between plasma- and tissue-based analyses was $64.5 \%$ in patients with lung metastasis alone $(n=31)$ indicating lower amount of ctDNA. Among the cases with lung metastasis alone, all plasma- and tissue-based analyses were perfectly concordant in cases with $\geq 20 \mathrm{~mm}$ of maximum lesion diameter or $\geq 10$ lesions.

CONCLUSION: The clinical validity of OnCoBEAM ${ }^{T M}$ RAS CRC kit was confirmed. Careful attention should be paid for mCRC patients with lung metastases alone having fewer metastases or smaller diameter lesions.

British Journal of Cancer (2019) 120:982-986; https://doi.org/10.1038/s41416-019-0457-y

\section{BACKGROUND}

Colorectal cancer (CRC) is the third most commonly diagnosed cancer and the fourth most common cause of cancer deaths worldwide. Although the epidermal growth factor receptor (EGFR) has become an important therapeutic target for CRC treatment, approximately $40 \%$ of patients with metastatic colorectal cancer (mCRC) have tumours with KRAS mutations, which are not expected to respond to anti-EGFR therapies. ${ }^{1,2}$ Furthermore, numerous comprehensive prospective or retrospective analysis of KRAS and NRAS codons $12,13,59,61,117$, and 146 demonstrated that patients with these mutations did not receive clinical benefits from anti-EGFR therapies. ${ }^{3}$

Cell-free DNA (cfDNA) is the natural DNA present in the cell-free fraction of the blood. Recent studies have suggested that genomic alterations in solid tumours can be characterised by studying the circulating-tumour DNA (ctDNA), which is the cfDNA released from cancer cells into the plasma. ${ }^{4}$ While ctDNA can exist in almost all patients with $\mathrm{mCRC}$, its low abundance requires highly sensitive techniques to detect the mutations presenting at low frequencies. The benefits of investigating the ctDNA are: provisions of a rapid genotype result with a streamlined clinical workflow and minimal patient invasiveness.

The OncoBEAM ${ }^{T M}$ RAS CRC Kit, which uses BEAMing digital PCR technology, is a CE-marked in vitro diagnostic based in Europe used for detecting RAS mutations in ctDNA derived from $m C R C$. Several prospective or retrospective studies to compare the RAS mutational status determined by BEAMing in plasma and the tissue reference method have been associated with concordance rates from $89.7 \%$ to $93.3 \% .^{5-7}$ Recently, García-Foncillas et al. demonstrated a concordance rate of 92.0\% ( $n=236)$ between the plasma-BEAMing and tissue reference method. The analysis of $8.0 \%$ of discordant cases suggested that the higher discordances were observed in the patient with lung metastasis alone. ${ }^{8}$

\footnotetext{
${ }^{1}$ Department of Gastroenterology and Gastrointestinal Oncology, National Cancer Center Hospital East, Kashiwa, Chiba, Japan; ${ }^{2}$ Department of Surgery, Kansai Rosai Hospital,

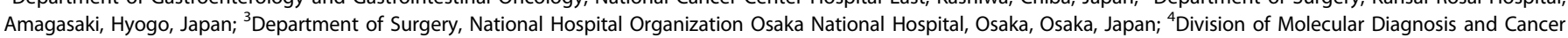
Prevention, Saitama Cancer Center, Ina, Saitama, Japan; ${ }^{5}$ Division of Gastroenterology, Chiba Cancer Center, Chiba, Chiba, Japan; ${ }^{6}$ Department of Gastrointestinal Medical

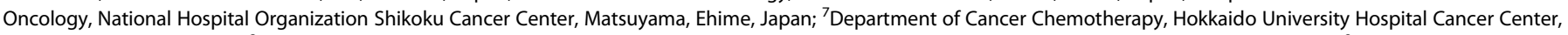

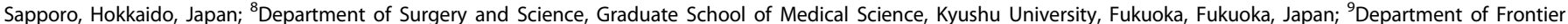

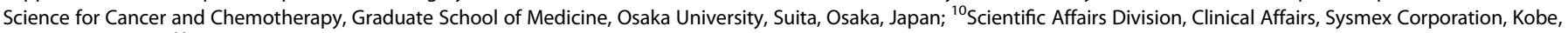
Hyogo, Japan and ${ }^{11}$ Department of Biostatistics, Yokohama City University School of Medicine, Yokohama, Kanagawa, Japan

Correspondence: Takayuki Yoshino (tyoshino@east.ncc.go.jp)

These co-first authors contributed equally as: Hideaki Bando and Yoshinori Kagawa
}

Received: 10 December 2018 Revised: 30 March 2019 Accepted: 4 April 2019

Published online: 24 April 2019 
Herein, we have conducted a prospective multicentre trial to investigate the concordance between the mutational status of RAS determined by plasma- and tissue-based BEAMing in patients with mCRC; we have also examined the characteristics of discordant cases.

\section{MATERIALS AND METHODS}

Study design and patients

This prospective study was carried out in eight Japanese medical centres from June 2017 to February 2018 (UMIN000023039). Patients with pathologically confirmed MCRC who were chemonaive or confirmed to have progressive disease (PD) without having initiated subsequent treatment and had an adequate archived formalin-fixed paraffin embedded (FFPE) tumour tissue specimen obtained within 5 years were enroled. Patients who had received prior treatment with anti-EGFR antibodies or regorafenib were excluded according to the possible appearance of acquired resistant mutations. ${ }^{9-11}$ This study was approved by the ethics committees at each institution (National Cancer Center Institutional Review Board, Kansai Rosai Hospital Review Board, Saitama Cancer Center Review Board, Chiba Cancer Center Review Board, National Hospital Organization Shikoku Cancer Center Review Board, Hokkaido University Review Board, Kyushu University Review Board, and Osaka University Review Board), and all patients provided written informed consent. All procedures related to the study were performed in accordance with the Helsinki Declaration.

\section{Procedures}

Plasma was obtained from $10 \mathrm{ml}$ of blood collected by Streck CellFree DNA BCT ${ }^{\circledR}$ Tube or BD Vacutainer ${ }^{\circledR}$ K2 EDTA Tube. FFPE tissues with $>30 \%$ tumour area were used for analysis. DNA was isolated from blood and FFPE by QIAamp Circulating Nucleic Acid Kit and QIAamp DNA FFPE, respectively. Genome equivalent (GE) of isolated DNA was quantified by LINE-1 quantitative real-time PCR assay as described previously. ${ }^{12}$

OncoBEAM ${ }^{\mathrm{TM}}$ RAS CRC Kit, which detects 34 mutations in KRAS/ NRAS codons $12,13,59,61,117$, and 146 , was used to analyse RAS mutations in plasma (plasma-BEAMing) at the Sysmex IMP laboratory, using the cut-off defined as the number of beads with amplified-mutant molecules specifically set per each codon (Kobe, Japan). FFPE tissue was used to analyse the 33 in KRAS/ NRAS codons 12,13,59, 61 (KRAS Q61R is excluded as compared with plasma-BEAMing), 117 , and 146 by BEAMing, as a reference method (tissue-BEAMing), using a $5 \%$ cut-off at Sysmex Inostics (Hamburg, Germany). MEBGEN ${ }^{T M}$ RASKET Kit (RASKET), ${ }^{13}$ which is approved as a companion diagnostic in Japan, was utilised to detect RAS mutations in FFPE tissue to validate the accuracy of tissue-BEAMing. Oncomine ${ }^{\mathrm{TM}}$ Colon cfDNA assays ${ }^{14}$ (plasma-NGS) using a cut-off of $0.05 \%$ were also utilise for reflex testing of all the cases where the plasma-BEAMing result was discordant with the tissue-BEAMing result. We also randomly sampled 100 cases from all the enroled cases and performed the plasma-NGS analysis for confirming the accuracy of plasma-BEAMing.

\section{Statistical analysis}

The primary analysis was to evaluate concordance between plasma- and tissue-RAS mutational status by BEAMing based on overall concordance rate, sensitivity (positive percent agreement), and specificity (negative percent agreement), where the evaluation was done for cases having both plasma- and tissue-testing results. Evaluation of plasma-BEAMing with reference to plasmaNGS, as well as that of tissue-BEAMing with reference to RASKET was also performed.

Factors associated with discordance were calculated using the univariate and multivariate logistic regression models. The multivariate analysis was performed using factors with a $P<0.1$ by the univariate analysis. Statistical analyses were performed using the $R$ i386 3.4.0 software.

\section{RESULTS}

Patient characteristics

A total of 350 patients with mCRC were initially enroled, 70 of whom were excluded for primary analysis due to the following reasons (Figure S1): lacking the qualified plasma or tissue availability $(n=$ $18)$, invalid results by tissue-BEAMing $(n=29)$, invalid results by plasma-BEAMing $(n=8)$, and conflicting exclusion criteria $(n=15)$. The remaining 280 patients were evaluated as a primary analysis set (Table 1). Most of the patients $(67 \%, 189 / 280)$ had recurrent diseases and more than half of the patients $(54 \%, 151 / 280)$ were chemo naive at the enrolment. Almost half of the patients (49\%, 138/280) had multiple sites of metastatic disease. In patients with only one metastatic site, the most frequent site was the liver $(27 \%, 76 / 280)$, followed by the lung $(11 \%, 31 / 280)$.

Concordance of RAS mutational status between plasma and tissue Overall, RAS mutations were detected in $44.3 \%$ of plasma samples and in $47.9 \%$ of tissue samples. The overall concordance between plasma- and tissue-based analyses was $86.4 \%(242 / 280)$, with a positive percent agreement of $82.1 \%(110 / 134)$ and negative

Table 1. Patient characteristics

\begin{tabular}{llll}
\hline Characteristics & & Primary analysis set $(N=280)$ & \% \\
\hline Age & Median, years [range] & $67[61-73]$ & 164 \\
Gender & Male & 91 & 39 \\
Newly diagnosed or relapsed & Newly diagnosis & 151 & 33 \\
Chemo naive & Yes & 91 & 34 \\
Location of primary tumour $^{\mathrm{a}}$ & Right-sided & 76 & 31 \\
Metastatic site & Liver alone & 27 \\
& Lung alone & 23 & 11 \\
& Lymph node alone & 12 \\
Sample collection interval from archived tissue to plasma & Other single organ & 138 \\
Source of tissue samples for test & Multiple sites & $11[1-61]$ \\
\hline
\end{tabular}


Table 2. Concordance between plasma and tissue-BEAMing

\begin{tabular}{|c|c|c|c|c|c|c|c|}
\hline & & \multicolumn{2}{|c|}{ Tissue-BEAMing } & Total & Concordance $\%[95 \% \mathrm{Cl}]$ & Sensitivity\% [95\% Cl] & Specificity\% $[95 \% \mathrm{Cl}]$ \\
\hline \multirow[t]{2}{*}{ Plasma-BEAMing } & MT & 110 & 14 & 124 & $86.4[81.9-90.2]$ & $82.1[74.5-88.2]$ & $90.4[84.4-94.7]$ \\
\hline & WT & 24 & 132 & 156 & & & \\
\hline Total & & 134 & 146 & 280 & & & \\
\hline
\end{tabular}

Table 3. Analytical accuracy of BEAMing; Plasma vs Plasma and Tissue vs Tissue

\begin{tabular}{|c|c|c|c|c|c|}
\hline & & \multicolumn{2}{|c|}{ Tissue-RASKET } & \multirow[t]{2}{*}{ Total } & \multirow{2}{*}{$\begin{array}{l}\text { Concordance\% } \\
{[95 \% \mathrm{Cl}]}\end{array}$} \\
\hline & & MT & WT & & \\
\hline \multirow[t]{2}{*}{ Tissue-BEAMing } & MT & 137 & 11 & 148 & 93.9 [90.5-96.3] \\
\hline & WT & 7 & 139 & 146 & \\
\hline \multirow[t]{3}{*}{ Total } & & 144 & 150 & 294 & \\
\hline & & \multicolumn{2}{|c|}{ Plasma-NGS } & Total & Concordance $\%$ \\
\hline & & MT & WT & & ] \\
\hline \multirow[t]{2}{*}{ Plasma-BEAMing } & MT & 48 & 3 & 51 & 96.0 [90.1-98.9] \\
\hline & WT & 1 & 48 & 49 & \\
\hline Total & & 49 & 51 & 100 & \\
\hline
\end{tabular}

percent agreement of $90.4 \%(132 / 146)$ (Table 2). Of the 38 discordant cases, we tested the 32 cases by plasma-NGS to investigate the reasons of discordances using different plasma assays, except for 6 cases who do not have enough samples for plasma-NGS. Patients with positive plasma-BEAMing and negative tissue-BEAMing results were observed in 14 cases, of which 6 were determined to be positive by plasma-NGS (table S1). On the other hand, patients with negative plasma-BEAMing and positive tissueBEAMing results were observed in 24 cases, of which 15 were confirmed to be negative by plasma-NGS (table S1). The concordance of the two different plasma methods was $96.0 \%$ (Table 3). Similarly, the concordance between tissue-BEAMing and RASKET using the same tissue samples was 93.9\% (Table 3).

Variables associated with discordance

We investigated the logistic regression analysis for identifying the factors associated with the discordance (Table 4). The most significant factor associated with discordance was lung metastasis alone, followed by sample collection interval from archived tissue to plasma. The multivariate analysis revealed that lung metastasis alone independently had a significant association with discordance.

Among the patients with lung metastasis alone, the overall agreement between the plasma- and tissue-based analyses was only $64.5 \%(20 / 31)$, while the overall concordance rate was $89.2 \%(222 / 249)$ in case of the patients excluding lung metastasis alone. Notably, the sensitivity of patients with lung metastasis alone was significantly lower than the cases excluding lung metastasis alone (41.2\%: 7/17 vs. $88.0 \%$ : 103/117) (Table 5).

Analysis of mutation allele frequency (MAF) and lesion volume A clear correlation between the metastatic site and mutation allele frequency (MAF) was observed. The median MAF [range] of the patients who had lung metastasis alone was $0.47 \%$ [0.03\%-2.21\%], which is significantly lower than patients who had other metastatic sites (Overall: $4.75 \%$ [0.01-59.76], Liver metastasis alone: $6.92 \%$ [0.01\%-44.09\%], LN metastasis alone: 3.56\% [0.36\%-21.21\%], Multiple metastasis: $8.19 \%$ [0.01\%-59.76\%]) (Fig. 1a). In order to find the trends of lower concordance of patients with lung metastasis alone $(n=31)$, we performed the post-hoc analysis, investigating the diameter and number of metastatic lesions. As shown in Fig. $1 \mathrm{~b}$, all discordant cases had the baseline longest diameter of lung lesion of smaller than $20 \mathrm{~mm}$ and less than 10 lesions (9/20, 11 cases were discordant.). Conversely, all cases were concordant with the baseline longest diameter of lung lesion equal to or larger than $20 \mathrm{~mm}$, or lesions equal to or more than 10 (11/11) (Fig. 1b).

\section{DISCUSSION}

This is the second clinical study to prospectively investigate the concordance of RAS mutational status between plasma- and tissueBEAMing and the first in Asian patients with mCRC. The concordance rate between plasma- and tissue-BEAMing was $86.4 \%$, which was comparable to those in previous studies $(89.7 \%-93.3 \%) .^{5-7}$ Of 38 discordant cases, 6 of 14 cases with positive plasma-BEAMing results and negative tissue-BEAMing results were plasma-NGS-positive (table S1). On the other hand, 15 of 24 cases with negative plasma-BEAMing and positive tissue-BEAMing results were plasma-NGS-negative (table S1). These results suggested that over half of the discordant cases (21/38) determined by plasmaBEAMing might be analytically accurate. In addition, a concordance rate of $96.0 \%$ between plasma-BEAMing and plasma-NGS was obtained, indicating an almost perfect agreement.

The plausible reason of discordance could be lower ctDNA shedding from tumours. The relevance between MAF burden and concordance were found as that median MAFs [range] with concordant cases and discordant cases were $7.33 \%[0.03 \%$ $59.76 \%]$ and $0.04 \%$ [0.01\%-30.25\%] $(p=0.001132$, wilcoxon rank sum test), respectively. Furthermore, in the logistic regression analysis, lung metastasis alone was the most significant factor associated with discordance, showing the lowest MAF $(0.47 \%)$, which is consistent with the finding reported by Vidal et al. describing lower MAF in patients with only lung metastatic involvement. $^{15}$ The concordance rate of the patients with lung metastasis alone was down to $64.5 \%$; especially, the sensitivity decreased to $41.2 \%$ (7/17), while the specificity was maintained at $92.9 \%$ (13/14). These results suggest that the plasma-BEAMing analyses for the cases with lung metastasis alone have a greater likelihood to show false negative results. Garcia-Foncillas et al. also reported that a low concordance rate was associated with the presence of lung metastasis alone. ${ }^{8}$ However, the population of those cases was considerably lower than that in our study [6.8\% (16/ $236)$ vs. $11.1 \%(31 / 280)]$, which was possibly associated with the slightly lower concordance rate in our study (86.4\%), compared to previous studies $(89.7 \%-93.3 \%) .^{5-7}$ Notably, we performed the posthoc analysis of cases with lung metastasis alone and identified that the maximum lesion diameter and number of lesions had an impact on the discordant results. Although a limited number of cases had been analysed, the longest diameter of lung lesion of $20 \mathrm{~mm}$, and lesions of 10 are specious cut-offs to discriminate the discordant cases. To furtherly investigate the optimal cut-off value of maximum 
Table 4. Comparison of baseline characteristics between concordant and discordant cases

\begin{tabular}{|c|c|c|c|c|c|}
\hline & & $\begin{array}{l}\text { Odds ratio } \\
\text { (Univariate) }\end{array}$ & $P$ value & $\begin{array}{l}\text { Odds ratio } \\
\text { (Multivariate) }\end{array}$ & $P$ value \\
\hline Age & - & 0.99 & 0.46 & - & - \\
\hline Newly diagnosed or relapsed & Relapsed & 0.51 & 0.11 & - & - \\
\hline Chemo naive & Yes & 1.2 & 0.6 & - & - \\
\hline Location of primary tumour & Right-sided & 1.26 & 0.58 & - & - \\
\hline & Lung alone & 0.22 & $<0.01$ & 0.24 & $<0.01$ \\
\hline & Lymph Node (LN) alone & 1.71 & 0.19 & - & - \\
\hline & Other single organ meta & 0.78 & 0.48 & - & - \\
\hline & Multiple metastasis & 1.4 & 0.74 & - & - \\
\hline Sample collection interval from archived tissue to plasma & - & 0.98 & 0.09 & 0.99 & 0.23 \\
\hline
\end{tabular}

Table 5. Concordance rate in cases with lung metastases alone or with excluding lung metastasis alone

\begin{tabular}{|c|c|c|c|c|c|c|c|}
\hline \multirow[t]{2}{*}{ Lung met. alone } & & \multicolumn{2}{|c|}{ Tissue-BEAMing } & \multirow[t]{2}{*}{ Total } & \multirow[t]{2}{*}{ Concordance\% $[95 \% \mathrm{Cl}]$} & \multirow[t]{2}{*}{ Sensitivity\% [95\% Cl] } & \multirow[t]{2}{*}{ Specificity\% [95\% Cl] } \\
\hline & & MT & WT & & & & \\
\hline Plasma-BEAMing & MT & 7 & 1 & 8 & $64.5[45.4-80.8]$ & $41.2[18.4-67.1]$ & $92.9[66.1-99.8]$ \\
\hline Total & 17 & 14 & 31 & & & & \\
\hline \multicolumn{2}{|c|}{ Excluding lung met. alone } & \multicolumn{2}{|c|}{ Tissue-BEAMing } & Total & Concordance $\%[95 \% \mathrm{Cl}]$ & Sensitivity\% [95\% Cl] & Specificity\% [95\% Cl] \\
\hline \multirow[t]{2}{*}{ Plasma-BEAMing } & MT & 103 & 13 & 116 & $89.2[84.6-92.7]$ & $88.0[80.7-93.3]$ & $90.2[83.7-94.7]$ \\
\hline & WT & 14 & 119 & 133 & & & \\
\hline Total & 117 & 132 & 249 & & & & \\
\hline
\end{tabular}

a

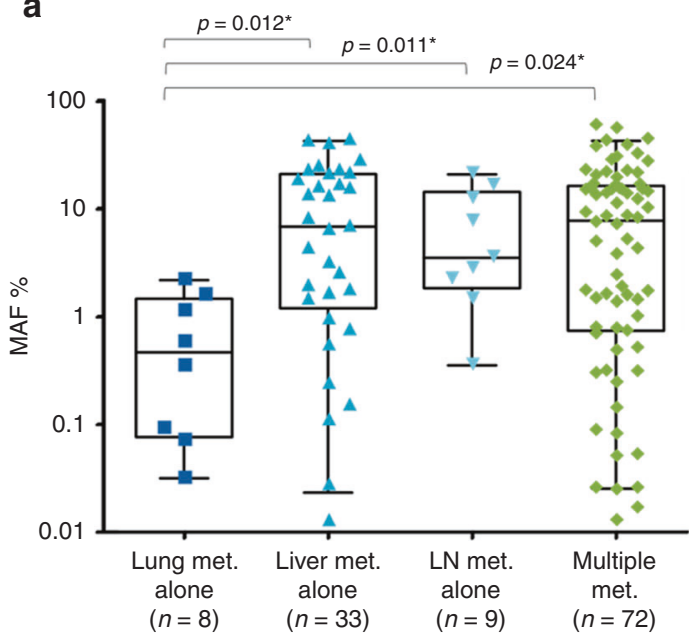

b

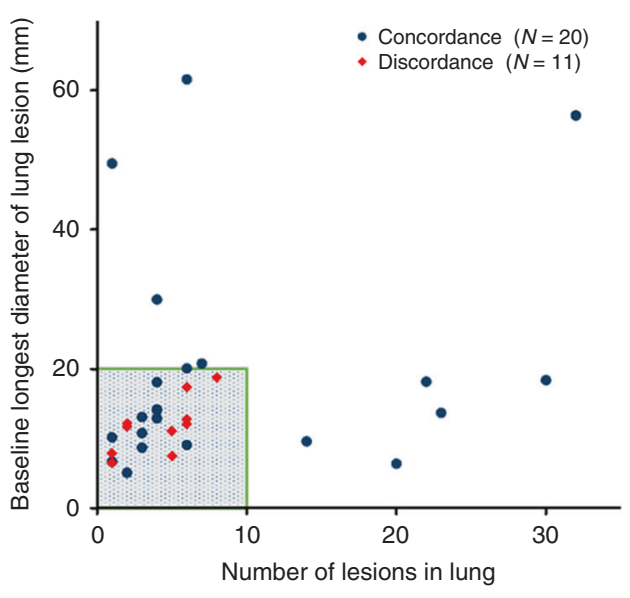

*derived from Steel test MAF: Mutant allele frequency

Fig. 1 Analysis of mutation allele frequency and lesion volume. Mutation allele frequency (MAF) in cases with metastatic site (a) and the longest diameter of lesion and number of lesions with concordant and discordant cases (b). * Derived from Steel test with control of group of lung metastasis alone 
diameter of lesion and number of lesions to predict concordant results in cases with lung metastasis alone or peritoneal metastasis alone, we are planning meta-analysis by combining our data with those in the previous western studies.

Other reasons for discordance could be clonal evolution and/or tumour heterogeneity. In the univariate logistic regression model, we found trends of the association of sample collection interval with discordance, suggesting clonal evolution due to longer intervals between plasma and tissue collections. Cases with heterogeneous tumours might also be included in plasmapositive and tissue-negative populations. Further investigation such as multiple-lesions or -times from tissue samples, and longitudinal assessment by plasma-NGS assessment to examine the mutational status of various genes, may lead the clarification of the tumour heterogeneity and clonal evolution. Additionally, investigation of efficacy with anti-EGFR antibodies in discordant cases, especially case with longer intervals between plasma and tissue collections, will establish the real value of OncoBEAM ${ }^{T M}$ RAS CRC Kit.

In conclusion, the reliability of plasma RAS mutational status determined by OncoBEAM ${ }^{\text {TM }}$ RAS CRC Kit was confirmed in Asian patients with $\mathrm{mCRC}$. Careful attention should be paid to interpret the results when we use the plasma-BEAMing for $\mathrm{mCRC}$ patients with lung metastases alone having fewer lung metastases as well as those with smaller diameter lesions.

\section{ACKNOWLEDGEMENTS}

We would like to express special thanks to all participating patients, their families, and all participating investigators.

\section{AUTHOR CONTRIBUTIONS}

All authors: Study concepts, Study design, Data acquisition, Manuscript editing, and Manuscript review. H.K. and T.Y.: Quality control of data and algorithms. H.B., Y.K., H.K., T.Y., T.Y.: Data analysis and interpretation. T.Y.: Statistical analysis. H.B., Y.K., H.K., T.Y., T.Y.: Manuscript preparation.

\section{ADDITIONAL INFORMATION}

Supplementary information is available for this paper at https://doi.org/10.1038/ s41416-019-0457-y.

Competing interests: H.B. reports research funding from AstraZeneca; T.D. reports research funding from Sanofi, Boehringer Ingelheim and MSD and honoraria from Chugai Pharmaceutical, Yakult Honsha, and Taiho Pharmaceutical; Y.K. reports the research funds from Merck Serono, Takeda, Chugai Pharmaceutical, Eli Lilly, Sanofi, Yakut Honsha, Taiho Pharmaceutical, Daiichi-Sankyo, Ono, MSD, Kyowa-kirin, Shionogi, Nipro, Dainihon, Eisai, BMS, Bayer Japan, and Pfizer; E.O. has received honoraria for lecturing from Taiho Pharmaceutical, Eli Lilly, Bayer Japan, Yakult Honsha, Merck Serono, Takeda, and Chugai Pharmaceutical; T.K. reports research funding from Yakult Honsha, Chugai Pharmaceutical, and Ono; H.K. reports employment by Sysmex Corporation during the conduct of the study; T.Y. reports research funding from Taiho Pharmaceutical and Takeda, honoraria from Taiho Pharmaceutical, Takeda, Chugai Pharmaceutical, and Boehringer Ingelheim, and advisory role from AstraZeneca, Daiichi-Sankyo, Gilead Sciences, Sysmex, and HUYA Bioscience, and reports research funding from GlaxoSmithKline and Nippon Boehringer Ingelheim and honoraria from Taiho Pharmaceutical, Eli Lilly Japan, and Chugai Pharmaceutical; and Y.K., T.K., T.N., and K.A. report no competing interest.

Ethics approval and consent to participate: This study was approved by the ethics committees at each institution, and all patients provided written informed consent. All procedures related to the study were performed in accordance with the Helsinki Declaration.

Data availability: All data generated or analysed during this study are included in this published article and its supplementary information files.
Publisher's note: Springer Nature remains neutral with regard to jurisdictional claims in published maps and institutional affiliations.

\section{REFERENCES}

1. Van Cutsem, E., Lenz, H. J., Köhne, C. H., Heinemann, V., Tejpar, S., Melezínek, I. et al. Fluorouracil, leucovorin, and irinotecan plus cetuximab treatment and RAS mutations in colorectal cancer. J. Clin. Oncol. 33, 692-700 (2015).

2. Bokemeyer, C., Köhne, C. H., Ciardiello, F., Lenz, H. J., Heinemann, V., Klinkhardt, U. et al. FOLFOX4 plus cetuximab treatment and RAS mutations in colorectal cancer. Eur. J. Cancer 51, 1243-1252 (2015).

3. Yoshino, T., Arnold, D., Taniguchi, H., Pentheroudakis, G., Yamazaki, K., Xu, R. H. et al. Pan-Asian adapted ESMO consensus guidelines for the management of patients with metastatic colorectal cancer: a JSMO-ESMO initiative endorsed by CSCO, KACO, MOS, SSO and TOS. Ann. Oncol. 29, 44-70 (2018).

4. Bettegowda, C., Sausen, M., Leary, R. J., Kinde, I., Wang, Y., Agrawal, N. et al. Detection of circulating tumor DNA in early- and late-stage human malignancies. Sci. Transl. Med 6, 224 ra24 (2014).

5. Schmiegel, W., Scott, R. J., Dooley, S., Lewis, W., Meldrum, C. J., Pockney, P. et al. Blood-based detection of RAS mutations to guide anti-EGFR therapy in colorectal cancer patients: concordance of results from circulating tumor DNA and tissuebased RAS testing. Mol. Oncol. 11, 208-219 (2017).

6. Grasselli, J., Elez, E., Caratù, G., Matito, J., Santos, C., Macarulla, T. et al. Concordance of blood- and tumor-based detection of RAS mutations to guide anti-EGFR therapy in metastatic colorectal cancer. Ann. Oncol. 28, 1294-1301 (2017).

7. García-Foncillas, J., Alba, E., Aranda, E., Díaz-Rubio, E., López-López, R., Tabernero, J. et al. Incorporating BEAMing technology as a liquid biopsy into clinical practice for the management of colorectal cancer patients: an expert taskforce review. Ann. Oncol. 28, 2943-2949 (2017).

8. García-Foncillas, J., Tabernero, J. M., Élez, E., Aranda, E., Benavides, M., Camps, C. et al, Prospective multicenter real-world RAS mutation comparison between OncoBEAM-based liquid biopsy and tissue analysis in metastatic colorectal cancer. Br. J. Cancer 119, 1464-1470 (in press).

9. Misale, S., Yaeger, R., Hobor, S., Scala, E., Janakiraman, M., Liska, D. et al. Emergence of KRAS mutations and acquired resistance to anti-EGFR therapy in colorectal cancer. Nature 28, 532-536 (2012).

10. Wong, A. L., Lim, J. S., Sinha, A., Gopinathan, A., Lim, R., Tan, C. S. et al. Tumour pharmacodynamics and circulating cell free DNA in patients with refractory colorectal carcinoma treated with regorafenib. J. Transl. Med. 13, 57 (2015).

11. Shitara, K., Yamanaka, T., Denda, T., Tsuji, Y., Shinozaki, K., Komatsu, Y. et al. REVERCE: A Randomized Phase II study of regorafenib followed by cetuximab versus the reverse sequence for previously treated metastatic colorectal cancer patients. Ann. Oncol. 30, 259-265 2019.

12. Rago, C., Huso, D. L., Diehl, F., Karim, B., Liu, G., Papadopoulos, N. et al. Serial assessment of human tumor burdens in mice by the analysis of circulating DNA. Cancer Res. 67, 9364-9370 (2007).

13. Yoshino, T., Muro, K., Yamaguchi, K., Nishina, T., Denda, T., Kudo, T. et al. Clinical validation of a multiplex kit for RAS mutations in colorectal cancer: results of the RASKET (RAS KEy Testing) prospective, multicenter study. EBioMedicine 2, 317-323 (2015)

14. Venkatesh, V., Laig, M., Varma, K., Li, Y., Bramlett, K., Dhingra, D. et al. Orthogonal validation of oncomine cfDNA panel data with digital PCR using TaqMan Rare Mutation Assays. Cancer Res. 77, 7392017.

15. Vidal, J., Muinelo, L., Dalmases, A., Jones, F., Edelstein, D., Iglesias, M. et al. Plasma ctDNA RAS mutation analysis for the diagnosis and treatment monitoring of metastatic colorectal cancer patients. Ann. Oncol. 28, 1325-1332 (2017).

Open Access This article is licensed under a Creative Commons Attribution 4.0 International License, which permits use, sharing, adaptation, distribution and reproduction in any medium or format, as long as you give appropriate credit to the original author(s) and the source, provide a link to the Creative Commons license, and indicate if changes were made. The images or other third party material in this article are included in the article's Creative Commons license, unless indicated otherwise in a credit line to the material. If material is not included in the article's Creative Commons license and your intended use is not permitted by statutory regulation or exceeds the permitted use, you will need to obtain permission directly from the copyright holder. To view a copy of this license, visit http://creativecommons. org/licenses/by/4.0/.

(c) The Author(s) 2019 